\title{
HPV-Mediated (p16-Positive) Oropharyngeal Cancer cN3 TNM Finding \\ v8
}

National Cancer Institute

\section{Source}

National Cancer Institute. HPV-Mediated (p16-Positive) Oropharyngeal Cancer CN3 TNM

Finding v8. NCI Thesaurus. Code C132875.

HPV-mediated (p16-positive) oropharyng eal cancer with lymph node(s) metastasis,

larger than $6 \mathrm{~cm}$. (from AJCC 8th Ed.) 